\title{
Increased CD27 expression in the skins and sera of patients with systemic sclerosis
}

\author{
Yuka Oshikawa, Takamitsu Makino, Miri Nakayama, Soichiro Sawamura, Katsunari Makino, \\ Ikko Kajihara, Jun Aoi, Shinichi Masuguchi, Satoshi Fukushima, Hironobu Ihn
}

Department of Dermatology and Plastic Surgery, Faculty of Life Sciences, Kumamoto University, Kumamoto, Japan.

SUMMARY Systemic sclerosis (SSc) is a kind of collagen disease and has an acquired autoimmune activation as represented by the production of autoantibodies. CD27 is a type I glycoprotein and a member of the tumor necrosis factor receptor family. It binds to the CD70 ligand, CD27-CD70 signaling is implicated in the development of various autoimmune diseases, but its role in the regulation of extracellular matrix expression and its contribution to the phenotype of SSc both remain to be elucidated. This study aimed to investigate the associations between CD27 and SSc in the skins and sera. Immunohistochemistry were performed to determine the expression of CD27 in the skin. Enzyme-linked immunosorbent assays were done to the sera of the 54 patients with SSc and 23 normal healthy controls. CD27 expression was significantly increased in the affected regions of the skin and the sera of patients of SSc. Thereafter, we evaluated the correlation between the serum soluble CD27 (sCD27) levels and the clinical symptoms. The study subjects with increased sCD27 levels had a significantly higher ratio of dcSSc and to showed higher modified Rodnan's total skin thickness scores (mRSS) than those with normal sCD27 levels. These results suggest that sCD27 levels might be useful for diagnosis of SSc and its severity.

Keywords CD27, tumor necrosis factor (TNF) receptor family, systemic sclerosis, fibrosis

\section{Introduction}

Systemic sclerosis (SSc) is an acquired autoimmune disorder, characterized by microvascular damage and excessive fibrosis of the skin and various internal organs. Based on the extent of skin fibrosis and the pattern of internal organ involvement, patients with SSc are commonly classified into either the diffuse cutaneous SSc (dcSSc) subset or the limited cutaneous $\mathrm{SSc}$ (1cSSc) subset (1). Although the pathogenesis of the disease is unclear, the clinical and pathological manifestations of SSc have been characterized as abnormalities in the innate and adaptive immune system that lead to the production of autoantibodies and cellleading to the accumulation of excessive collagen and other extracellular matrices in the skin, blood vessels, and internal organs (2). Fibrosis is a major contributor to the high level of morbidity and mortality in SSc patients, and it is believed that the expression levels of proinflammatory chemokines, cytokines, and connective tissue growth factors are elevated, which leads to the activation of fibroblasts and the abnormal accumulation of the extracellular matrix. This results in progressive endothelial damage, edema, and sclerosis of the skin (3).
CD27 is a type I glycoprotein that is expressed on the majority of $\mathrm{T}$ cells and some B cells, and it is a member of the tumor necrosis factor (TNF) receptor family with unique cysteine-rich motifs (4). In subsequent studies, it was revealed that CD27 is expressed on CD4 and CD8 T lymphocytes, NK cells, and hematopoietic stem cells $(5,6)$. The activation of $\mathrm{T}$ cells can also lead to the shedding of CD27 from the cell surface, resulting in the secretion of soluble CD27 (sCD27) (7). On the contrary, CD27 is not expressed by naïve $\mathrm{B}$ lymphocytes, but is upregulated in activated and antigen-experienced B lymphocytes (8). CD27 is also uniformly found on memory B cells (9). It binds to the ligand CD70 and transduces signals that lead to the activation of nuclear factor-kappa $\mathrm{B}(\mathrm{NF}-\kappa \mathrm{B})$ and mitogen-activated protein kinase 8 (MAPK) / c-jun N-terminal kinase(JNK). Adaptor proteins, such as TNF receptor-associated factor (TRAF) 2 and TRAF5, which have been shown to mediate the signaling process of this receptor. The CD27-binding protein (SIVA), a pro-apoptotic protein, can bind to this receptor, and then regulates many cellular processes, including cell proliferation, differentiation, and survival $(10,11)$. Thus, a key biological role for the interactions of CD27-CD70 
in $\mathrm{T}$ cell priming and in the subsequent promotion of their survival, which results in the formation of effector and memory T cells, has been well documented (12,13). In the B cell compartment, CD27-CD70 interactions are important for $\mathrm{T}$ cell-dependent antibody production by promoting B cell activation, germinal center formation, expansion of B cells, and differentiation into plasma cells, and by enhancing immunoglobulin production (14-16). However, the involvement of CD27 in the pathogenesis of SSc remains unknown.

In this study, we examined the expression of CD27 in the skin of SSc patients by immunohistochemistry. The amount of CD27 was measured in dcSSc, lcSSc patients and healthy controls. Furthermore, we examined the serum levels of sCD27 in SSc patients by enzyme-linked immunosorbent assay (ELISA). We evaluated the correlation between the serum SCD27 levels and clinical symptoms in patients with SSc.

\section{Materials and Methods}

\subsection{Patients}

The study was approved by the Ethical Committee of the Kumamoto University School of Medicine and was carried out according to the guidelines set by the Institutional Review Board. We obtained tissue samples from 8 patients with SSc and serum samples from 54 patients with SSc. All patients with SSc fulfilled the new criteria proposed by the American College of Rheumatology/European League against Rheumatism (17), and were grouped according to the classification system proposed by LeRoy et al. (18). Of the 8 patients, 5 patients had diffuse cutaneous SSc $(\mathrm{dcSSc})$, and 3 patients had limited cutaneous SSc ( $1 \mathrm{cSSc}$ ), as described above. Skin biopsy specimens for the SSc patients were obtained from the involved skin. Control skin samples were obtained from the routinely discarded skin of healthy human subjects undergoing skin grafts. The samples were fixed in formalin immediately after removal and were then embedded in paraffin. On the contrary, the serum samples were obtained from 23 patients with deSSc and 31 patients with lcSSc. Control serum samples were also collected from 18 healthy ageand sex-matched volunteers. All serum samples were stored at $-80^{\circ} \mathrm{C}$ prior to use.

Institutional review board approval and written informed consent were obtained before the patients and healthy volunteers entered in this study, according to the Declaration of Helsinki.

\subsection{Immunohistochemical (IHC) staining and IHC evaluation}

Immunohistochemical analysis was performed on $4 \mu \mathrm{m}$ sections of formalin-fixed, paraffin-embedded tissue. The sections were deparaffinized with Clear
Plus ${ }^{\circledR}$ (Falma, Tokyo, Japan) and were subsequently rehydrated with ethanol. Antigen retrieval was performed in a microwave oven with a citric acid (pH 6.0) buffer. Endogenous peroxidase activity was blocked by incubation with $1.0 \%$ hydrogen peroxidase. After the addition of normal goat serum (FUJIFILM Wako Pure Chemical, Osaka, Japan), a monoclonal rabbit anti-CD27 antibody (Abcam, Cambridge, UK) (1:250 dilution) was used as the primary antibody, and tissue samples were incubated with this antibody overnight at $4^{\circ} \mathrm{C}$. These sections were further incubated with Histofine ${ }^{\circledR}$ Simple Stain MAX-PO(R) (Nichirei Biosciences, Tokyo, Japan) for $30 \mathrm{~min}$. Finally, the chromogen was 3,3'-diaminobenzidine, and the sections were counterstained with Mayer's hematoxylin to facilitate the recognition of structures.

\subsection{Soluble CD27 measurement}

Serum levels of sCD27 were measured with a specific ELISA kit (Human CD27/, Abnova, Taipei, Taiwan). Briefly, human CD27 capture antibodies were precoated onto microtiter wells. Aliquots of serum were added to each well, followed by the peroxidase-conjugated antibodies for CD27. The color was developed with hydrogen peroxidase and tetramethylbenzidine peroxidase, and the absorbance at $450 \mathrm{~nm}$ was measured. Wavelength correction was performed based on the absorbance of a blank well at $450 \mathrm{~nm}$. The concentration of sCD27 in each sample was determined by interpolation from a standard curve.

\subsection{Statistical analysis}

Statistical analysis was carried out with the MannWhitney test for the comparison of the median, and Fisher's exact probability test was used for the analysis of the frequency. A $p$ value of less than 0.05 was considered significant.

\section{Results and Discussion}

3.1. Abundant expression of CD27-positive lymphocytes in the skin of SSc patients

First, we attempted to evaluate the expression pattern of CD27 in the involved skin of SSc patients. Skin tissue samples were obtained from 5 patients with dcSSc and 3 with lcSSc. The immunohistochemistry of CD27 was examined in the skin tissues from patients with dcSSc and lcSSc. CD27 was found on the surface of lymphocytes around the capillaries in the reticular dermis. Both lymphocytes and CD27-positive lymphocytes were more abundant in the skin of patients with deSSc (Figure 1A and 1B). Then, the ratio of CD27-positive lymphocytes to overall lymphocytes was calculated for each of five high-power fields, 
respectively. The ratios of CD27-positive cells were significantly higher in the skin of the dcSSc patients than in the lcSSc patients $(32.2 \%$ vs. $19.7 \%, p<0.05)$, as shown in Figure 2. Jacobi AM et al. reported that CD27-positive plasma cells were elevated in the PBMC of patients with SLE (19). However, whether CD27 is involved in the pathogenesis of skin sclerosis in SSc patients remains to be elucidated. Our results suggest that CD27 is activated in the skins of dcSSc patients and may be useful for the diagnosis.
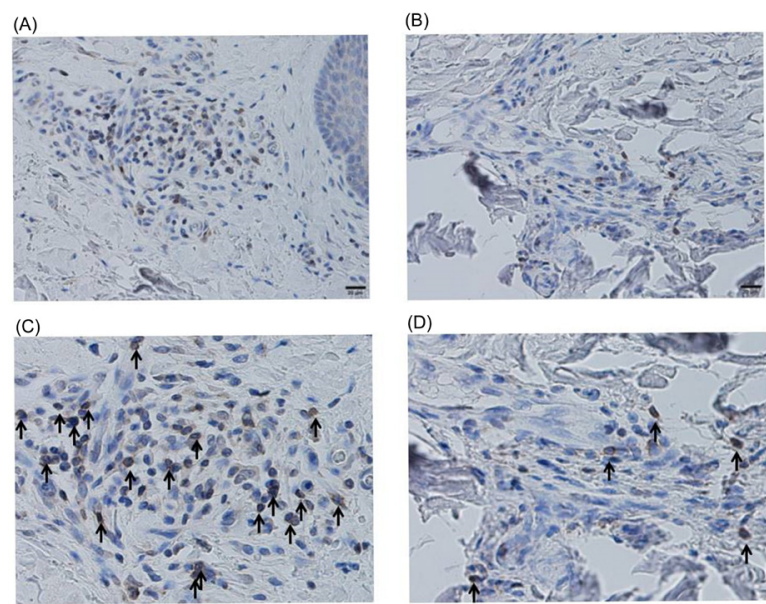

Figure 1. Abundant expression of CD27-positive lymphocytes in the skin of SSc patients. Paraffin sections were subjected to the immunohistochemical analyses. Both the lymphocytes and CD27positive lymphocytes were abundant in the skin of patients with dcSSc $(\mathbf{A}, \mathbf{C})$ compared to lcSSc (B,D). Black arrow indicates CD27positive cells in high-power fields. It shows at Low-power field $(\times$ 100) $(\mathbf{A}, \mathbf{C})$ and high-power field $(\times 200)(\mathbf{B}, \mathbf{D})$.

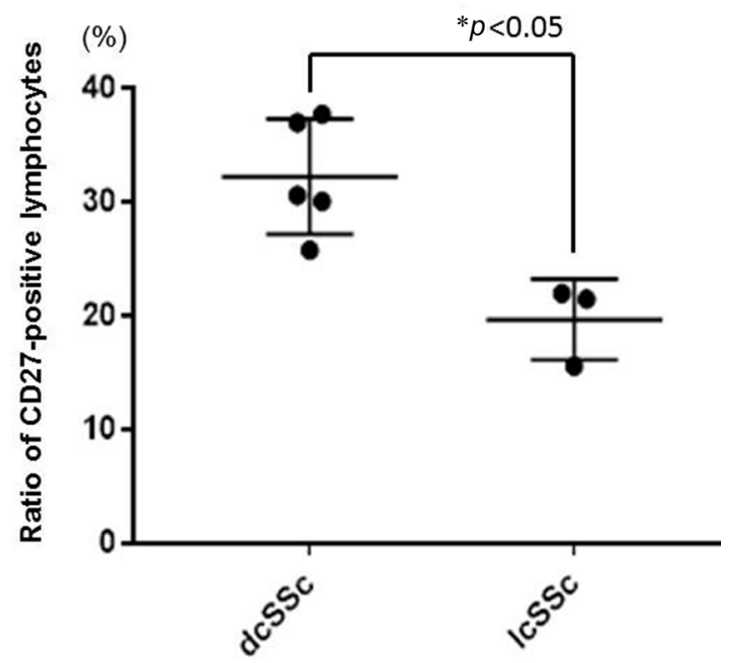

Figure 2. High ratios of CD27-positive lymphocytes in deSSc patients. The samples were prepared from skin tissues obtained from patients with dcSSc $(n=5)$ and those with $\operatorname{lcSSc}(n=3)$. The ratio of CD27-positive cells to overall lymphocytes was calculated for each of the five high-power fields from each sample, respectively. The ratios of CD27-positive cells in dcSSc patients were significantly higher than those in lcSSc patients (32.2\% vs. 19.7\%). Bars indicate means (SD). $p$ values less than 0.05 are interpreted as significant.
3.2. Elevated soluble CD27 levels in the sera of SSc patients

Next, we examined the expression of sCD27 in serum by sandwich ELISA, as described in the methods section. Serum samples were obtained from 54 patients with SSc (23 dcSSc and $31 \mathrm{lcSSc}$ ). Samples were also obtained from 18 healthy control subjects. The serum levels of sCD27 in patients with dcSSc and lcSSc and in the healthy control subjects are shown in Figure 3. Patients with deSSc had significantly higher sCD27 levels than those with $\mathrm{lcSSc}(4,319 \pm 1,701 \mathrm{pg} / \mathrm{mL} v s$. $3,305 \pm 1,189 \mathrm{pg} / \mathrm{mL}, p<0.05)$. Additionally, the $\mathrm{lcSSc}$ patients had significantly higher sCD27 levels than the healthy controls $\operatorname{did}(3,305 \pm 1,189 \mathrm{pg} / \mathrm{mL}$ vs. $1,781 \pm$ $593 \mathrm{pg} / \mathrm{mL}, p<0.05)$. These results suggest that CD27 is also activated in the sera of SSc patients, particularly in those of dcSSc.

\subsection{Systemic activation of CD27 in SSc patients}

According to our results as described above, CD27 was activated not only in the skin tissues of patients with SSc, but also in the sera of patients with SSc. These results suggest that the systemic activation of CD27 is associated with the pathogenesis of SSc. In SLE patients, Font $\mathrm{J}$ et al. reported that the serum levels of sCD27 were elevated and correlated with disease activity measures (20). However, the efficacy of CD27 as a biomarker for the diagnosis or prognosis prediction of SSc is still unknown.

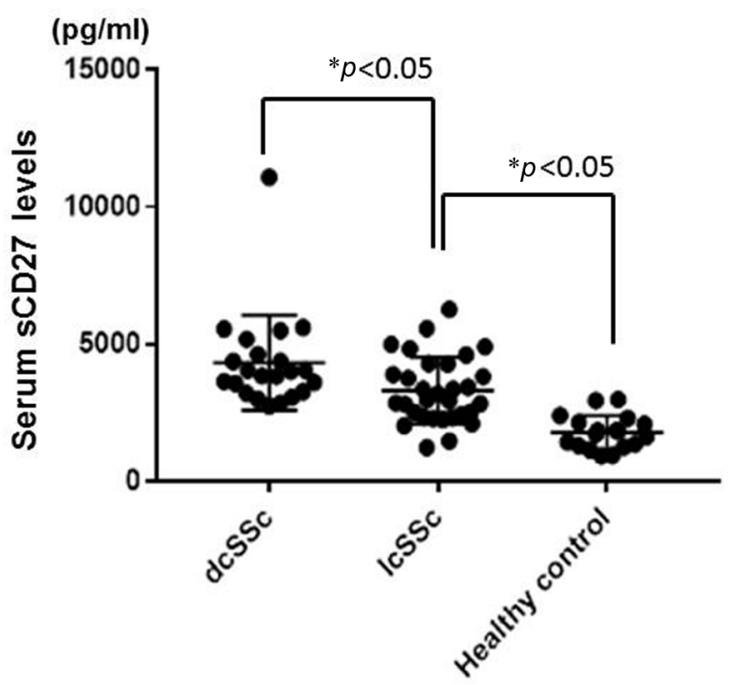

Figure 3. Elevated soluble CD27 levels in the sera of SSc patients. Serum CD27 levels were measured with ELISA kits. Patients with dcSSc had significantly higher sCD27 levels than the patients with lcSSc $(4,464 \mathrm{pg} / \mathrm{mL}$ vs. 3,305 pg/mL, $p<0.05)$. Additionally, the lcSSc patients had significantly higher sCD27 levels than the healthy controls did $(3,305 \mathrm{pg} / \mathrm{mL}$ vs. $1,781 \mathrm{pg} / \mathrm{mL}, p<0.05)$. Bars show means (SD). $p$ values less than 0.05 are interpreted as significant. 
Then, we evaluated the correlation between sCD27 and the clinical symptoms. We divided the SSc patients into two groups. The increased sCD27 group was defined as having much higher sCD27 levels than the mean $+2 \mathrm{SD}$ of the healthy controls, while the normal sCD27 group was defined as having less than the mean $+2 \mathrm{SD}$. The ratio of the dcSSc patients and modified Rodnan's total skin thickness scores (mRSS) were statistically significantly higher in the increased sCD27 group, whereas the number of patients with calcinosis was larger in normal sCD27 group. There were no statistically significant differences in the sex, age of onset, disease duration, other clinical symptoms, or organ involvement (Table 1). These results were consistent with those of the immunohistochemical analysis of the skin tissues and suggested that CD27 could play a key role in skin fibrosis.

CD27-CD70 is co-stimulatory molecule, the signaling of which leads to the activation of $\mathrm{T}$ cells and $\mathrm{B}$ cells and induces the proliferation of plasma cells and the production of immunoglobulin G (21). Furthermore, CD27-CD70 signaling is considered to trigger the pathophysiology of autoimmune diseases. In SSc patients, Jiang $\mathrm{H}$, et al. reported that the proportion of CD4+ T cells expressing CD70 was significantly increased compared to the controls (22). CD70 was significantly more expressed in CD4 T cells of patients with RA than in those of age-matched healthy controls (23). Oflazoglu E, et al. reported the efficacy of treatment of collagen-induced arthritis (CIA) with an anti-CD70 antibody in a murine model. Treatment

Table 1. Correlation of serum sCD27 with clinical symptoms

\begin{tabular}{|c|c|c|}
\hline sCD27 & Increased $(n=36)$ & Normal $(n=19)$ \\
\hline Male:Female & $8: 28$ & $0: 19$ \\
\hline Age (average) & $67.2(31-84)$ & $60.6(33-84)$ \\
\hline Disease duration (year) & 6.51 & 5.53 \\
\hline Diffuse:limited & $21: 15^{*}$ & $3: 16$ \\
\hline topo1:ACA:RNP & $12: 13: 4$ & $5: 8: 1$ \\
\hline mRSS & $14(1-41)^{*}$ & $8.5(0-34)$ \\
\hline \multicolumn{3}{|l|}{ Clinical symptoms (\%) } \\
\hline Raynaud's phenomenon & 82.4 & 93.3 \\
\hline Pitting scar & 38.7 & 42.9 \\
\hline Didital ulcer & 38.2 & 18.8 \\
\hline Nailfold bleeding & 50.0 & 61.5 \\
\hline Telangiectasia & 36.0 & 40.0 \\
\hline Contracture of phalanges & 87.1 & 84.6 \\
\hline Caltinosis & $4.55^{*}$ & 25.0 \\
\hline Sicca sumptoms & 64.3 & 57.1 \\
\hline \multicolumn{3}{|l|}{ Organ involvement (\%) } \\
\hline ILD & 52.8 & 41.2 \\
\hline Heart & 51.4 & 40.0 \\
\hline Esophagus & 35.3 & 28.6 \\
\hline Liver & 14.3 & 12.5 \\
\hline Kidney & 8.57 & 0.00 \\
\hline Joint & 24.0 & 50.0 \\
\hline Thyroiditis & 12.1 & 6.25 \\
\hline
\end{tabular}

ACA, anti-centromere antibody; ILD, intestinal lung disease; mRSS, modified Rodnan total skin thickness score; RNP, anti-U1 RNP antibody; topo1, anti-topoisomerase 1 antibody. ${ }^{*} p<0.05$. with an anti-CD70 antibody, both before onset and after established disease in a CIA model resulted in significant improvements in the disease activity and reduction in the production of autoantibodies (24). However, it remains to be seen whether a therapeutic blockade of CD70 might be a useful approach for the treatment of RA or other inflammatory arthritides, or whether CD27-CD70 signaling is involved in the pathogenesis of SSc. Clinical trials of an anti-CD70 antibody for the treatment of malignant tumours have been ongoing. Similarly, inhibition of CD27-70 signaling might be a target for the treatment of SSc.

In conclusion, the CD27 expression levels in skin tissues and sCD27 levels in serum samples are useful as diagnostic markers for SSc. Furthermore, they have the possibility of being markers for the disease activity or for the prediction of the response to treatment for SSc. In this study, only a small number of patients were evaluated, due to the rarity of SSc; large-scale studies on CD27 and CD70 in patients with SSc should be conducted in the future.

\section{Acknowledgements}

This research was supported by AMED under Grant Number 18ek0109328h0001. The funders had no role in study design, date collection and analysis, decision to publish, or preparation of the manuscript.

\section{References}

1. Wollheim FA. Classification of systemic sclerosis. Visions and reality. Rheumatology (Oxford). 2005; 44:1212-1216.

2. Jinnin M. Mechanisms of skin fibrosis in systemic sclerosis. J Dermatol. 2010; 37:11-25.

3. Nikpour M, Stevens WM, Herrick AL, and Proudman SM. Epidemiology of systemic sclerosis. Best Pract Res Clin Rheumatol. 2010; 24:857-869.

4. Maurer D, Fischer GF, Fae I, Majdic O, Stuhlmeier K, Von Jeney N, Holter W, Knapp W. IgM and IgG but not cytokine secretion is restricted to the $\mathrm{CD} 27+\mathrm{B}$ lymphocyte subset. J Immunol. 1992; 148:3700-3705.

5. Tesselaar K, Xiao Y, Arens R, van Schijndel GM, Schuurhuis DH, Mebius RE, Borst J, van Lier RA. Expression of the murine $\mathrm{CD} 27$ ligand $\mathrm{CD} 70$ in vitro and in vivo. J Immunol. 2003; 170:33-40.

6. Han BK, Olsen NJ, and Bottaro A. The CD27-CD70 pathway and pathogenesis of autoimmune disease. Semin Arthritis Rheum. 2016; 45:496-501.

7. Hintzen RQ, van Lier RA, Kuijpers KC, Baars PA, Schaasberg W, Lucas CJ, Polman CH. Elevated levels of a soluble form of the $\mathrm{T}$ cell activation antigen CD27 in cerebrospinal fluid of multiple sclerosis patients. J Neuroimmunol. 1991; 35:211-217.

8. Loenen WA, De Vries E, Gravestein LA, Hintzen RQ, Van Lier RA, and Borst J. The CD27 membrane receptor, a lymphocyte-specific member of the nerve growth factor receptor family, gives rise to a soluble form by protein processing that does not involve receptor endocytosis. Eur J Immunol. 1992; 22:447-455. 
9. Klein U, Rajewsky K, and Küppers R. Human immunoglobulin (Ig) $\mathrm{M}+\operatorname{IgD}+$ peripheral blood $\mathrm{B}$ cells expressing the CD27 cell surface antigen carry somatically mutated variable region genes: $\mathrm{CD} 27$ as a general marker for somatically mutated (memory) B cells. J Exp Med. 1998; 188:1679-1689.

10. Akiba H, Nakano H, Nishinaka S, Shindo M, Kobata T, Atsuta M, Morimoto C, Ware CF, Malinin NL, Wallach D, Yagita H, Okumura K. CD27, a member of the tumor necrosis factor receptor superfamily, activates NF-kappaB and stress-activated protein kinase/c-Jun N-terminal kinase via TRAF2, TRAF5, and NF-kappaB-inducing kinase. J Biol Chem. 1998; 273:13353-13358.

11. Ramakrishnan P, Wang W, and Wallach D. Receptorspecific signaling for both the alternative and the canonical NF-kappaB activation pathways by NFkappaB-inducing kinase. Immunity. 2004; 21:477-489.

12. Lens SM, Tesselaar K, van Oers MH, and van Lier RA. Control of lymphocyte function through CD27-CD70 interactions. Semin Immunol. 1998; 10:491-499.

13. Sugita K, Hirose T, Rothstein DM, Donahue C, Schlossman SF, and Morimoto C. CD27, a member of the nerve growth factor receptor family, is preferentially expressed on CD45RA+ CD4 T cell clones and involved in distinct immunoregulatory functions. J Immunol. 1992; 149:3208-3216.

14. Jacquot S, Kobata $\mathrm{T}$, Iwata $\mathrm{S}$, Morimoto $\mathrm{C}$, and Schlossman SF. CD154/CD40 and CD70/CD27 interactions have different and sequential functions in $\mathrm{T}$ cell-dependent B cell responses: enhancement of plasma cell differentiation by CD27 signaling. J Immunol. 1997; 159:2652-2657.

15. Xiao Y, Hendriks J, Langerak P, Jacobs H, and Borst J. CD27 is acquired by primed B cells at the centroblast stage and promotes germinal center formation. J Immunol. 2004; 172:7432-7441.

16. Agematsu K, Nagumo H, Oguchi Y, Nakazawa T, Fukushima K, Yasui K, Ito S, Kobata T, Morimoto C, Komiyama A. Generation of plasma cells from peripheral blood memory B cells: synergistic effect of interleukin-10 and CD27/CD70 interaction. Blood. 1998; 91:173-180.

17. van den Hoogen F, Khanna D, Fransen J, et al. 2013 classification criteria for systemic sclerosis: an American College of Rheumatology/European League against
Rheumatism collaborative initiative. Arthritis Rheum. 2013; 65:2737-2747.

18. LeRoy EC, Black C, Fleischmajer R, Jablonska S, Krieg T, Medsger TAJr, Rowell N, Wollheim F. Scleroderma (systemic sclerosis): classification, subsets and pathogenesis. J Rheumatol. 1988; 15:202-205.

19. Jacobi AM, Odendahl M, Reiter K, Bruns A, Burmester GR, Radbruch A, Valet G, Lipsky PE, Dörner T. Correlation between circulating CD27high plasma cells and disease activity in patients with systemic lupus erythematosus. Arthritis Rheum. 2003; 48:1332-1342.

20. Font J, Pallares L, Martorell J, Martinez E, Gaya A, Vives J, Ingelmo M. Elevated soluble CD27 levels in serum of patients with systemic lupus erythematosus. Clin Immunol Immunopathol. 1996; 81:239-243.

21. Denoeud J, Moser M. Role of CD27/CD70 pathway of activation in immunity and tolerance. J Leukoc Biol. 2011; 89:195-203.

22. Jiang H, Xiao R, Lian X, Kanekura T, Luo Y, Yin Y, Zhang G, Yang Y, Wang Y, Zhao M, Lu Q. Demethylation of TNFSF7 contributes to CD70 overexpression in CD4+ $\mathrm{T}$ cells from patients with systemic sclerosis. Clin Immunol. 2012; 143:39-44.

23. Lee WW, Yang ZZ, Li G, Weyand CM, Goronzy JJ. Unchecked CD70 expression on T cells lowers threshold for $\mathrm{T}$ cell activation in rheumatoid arthritis. J Immunol. 2007; 179:2609-2615.

24. Oflazoglu E, Boursalian TE, Zeng W, Edwards AC, Duniho S, McEarchern JA, Law CL, Gerber HP, Grewal IS. Blocking of CD27-CD70 pathway by anti-CD70 antibody ameliorates joint disease in murine collageninduced arthritis. J Immunol. 2009; 183:3770-3777.

Received April 12, 2020; Revised May 6, 2020; Accepted May 13, 2020

*Address correspondence to:

Takamitsu Makino, Department of Dermatology and Plastic Surgery, Faculty of Life Sciences, Kumamoto University, 1-11 Honjo, Kumamoto 860-8556, Japan.

E-mail: makinoderma@gmail.com

Released online in J-STAGE as advance publication May 15, 2020 . 